\title{
Neutrophil CD64 Expression as a Diagnostic Marker in Patients Hospitalized with Exacerbations of COPD: A Prospective Observational Study
}

\author{
Elena Titova ${ }^{1,2}$ - Marthe Wedø Aune ${ }^{3}$ Kristin Fonn ${ }^{3}$ - Anne Hildur Henriksen ${ }^{1,2}$ • \\ Arne Åsberg ${ }^{4}$
}

Received: 25 March 2015 / Accepted: 1 July 2015/Published online: 15 July 2015

(C) The Author(s) 2015. This article is published with open access at Springerlink.com

\begin{abstract}
Purpose The expression of the neutrophil high-affinity Fc-gamma receptor (CD64) can be used as a diagnostic marker for bacterial infection and sepsis. The aims of this study were to determine the diagnostic accuracy of CD64 compared to C-reactive protein (CRP) and white blood cell count (WBC) in patients hospitalized with acute exacerbations of COPD (AECOPD) and to investigate the kinetics of CD64 expression.

Methods The present study is a prospective, single-centre observation study. Blood samples were collected from patients hospitalized with AECOPD at admission and after 6, 24 and $48 \mathrm{~h}$. Retrospective reviews on the patients' medical records were performed blinded to the CD64
\end{abstract}

Elena Titova

elena.titova@ntnu.no

Marthe Wedø Aune

Marthe.Wedo.Aune@stolav.no

Kristin Fonn

Kristin.Fonn@stolav.no

Anne Hildur Henriksen

Anne.H.Henriksen@ntnu.no

Arne Åsberg

Arne.Aasberg@stolav.no

1 Department of Circulation and Medical Imaging, Norwegian University of Science and Technology, 7006 Trondheim, Norway

2 Department of Thoracic Medicine, Trondheim University Hospital, 7006 Trondheim, Norway

3 Department of Immunology and Transfusion Medicine, Trondheim University Hospital, 7006 Trondheim, Norway

4 Department of Clinical Chemistry, Trondheim University Hospital, Trondheim 7006, Norway results. The CD64 was measured using the Leuko64 kit from Trillium Diagnostics, LLC (Maine, USA) with the CELL-DYN Sapphire Haematology System (Abbott Laboratories, Illinois, USA). Diagnostic accuracy of the CD64, $\mathrm{CRP}$ and WBC was compared using a receiver operating characteristic (ROC) curve analysis.

Results A total of 113 patients were included. Thirty-six patients (32\%) had pulmonary infiltrate on chest X-ray at admission (PI). The CD64 was higher in samples from patients with AECOPD and PI than those without PI at admission (median 1.25 vs. $0.60, p=0.002$ ) and during $48 \mathrm{~h}$ of follow-up. The area under the ROC curve of CD64, CRP and WBC was $0.69,0.73$ and 0.64 , respectively, ( $p=0.42$ for the test of difference).

Conclusion Neutrophil CD64 expression has about the same diagnostic accuracy as CRP in diagnosing pneumonia in patients hospitalized with AECOPD, but does not add to the diagnostic accuracy of CRP and WBC count.

Keywords COPD exacerbation - Infection - Biomarkers · Neutrophil CD64 expression · Diagnostic accuracy

$\begin{array}{ll}\text { Abbreviations } & \\ \text { CD64 } & \begin{array}{l}\text { Neutrophil high-affinity Fc-gamma } \\ \text { receptor expression }\end{array} \\ \text { COPD } & \text { Chronic obstructive pulmonary disease } \\ \text { AECOPD } & \begin{array}{l}\text { Acute exacerbations of COPD } \\ \text { CRP }\end{array} \\ \text { C-reactive protein } \\ \text { WBC } & \begin{array}{l}\text { White blood cell } \\ \text { PI }\end{array} \\ \text { Pulmonary infiltrate on chest X-ray at } \\ \text { p-AECOPD } & \text { admission } \\ \text { np-AECOPD } & \text { AECOPD with PI } \\ \text { ROC } & \text { Receiver operating characteristic } \\ \text { DTM } & \text { Department of Thoracic Medicine }\end{array}$




$\begin{array}{ll}\text { TUH } & \text { Trondheim University Hospital } \\ \text { FEV }_{1} & \begin{array}{l}\text { Forced expired volume (litres) in one } \\ \text { second }\end{array} \\ \text { FEV }_{1} \% & \text { FEV }_{1} \text { percent of predicted value } \\ \text { FVC } & \text { Forced vital capacity (litres) } \\ \text { FVC\% } & \text { FVC percent of predicted value } \\ \text { FEV } / \text { FVC } & \text { FEV1 expressed as a proportion of FVC } \\ \text { ICS } & \text { Inhaled corticosteroids } \\ \text { LABA } & \text { Long-acting beta-adrenoceptor agonist }\end{array}$

\section{Introduction}

Chronic obstructive pulmonary disease (COPD) is characterized by a persistent airflow limitation that is usually progressive and associated with an enhanced chronic inflammatory response in the airways and the lungs to noxious particles or gases. COPD is a leading cause of morbidity and mortality worldwide, and represent an economic and social burden that is both substantial and increasing [1].

Exacerbations of COPD (AECOPD), episodes of an acute increase in respiratory symptoms, are associated with increased airway and systemic inflammation [2, 3]. AECOPD are associated with significant morbidity and an impaired quality of life, and are the primary drivers of hospital admissions $[4,5]$.

About half of the AECOPD are triggered by bacterial and viral infections, but pollution can also contribute to the beginning of an exacerbation [6-9]. AECOPD are heterogeneous events, and there are no specific biomarkers to determine their causation [10].

Physicians' decisions would be more precise if they could be guided by the results of accurate biomarkers, which might not only differentiate stable diseases from exacerbations, but also predict the severity of such events [11]. After the first publications from Davis and colleagues in 1995, about the diagnostic potential of neutrophil CD64 [12] and from Bakke and colleagues, who for the first time reported statistical measures for the performance of CD64 as a diagnostic test in 2001 [13], a number of publications in the field have shown that the neutrophil CD64 expression (CD64) could be a useful diagnostic cell-based parameter of systemic bacterial infection particularly in relation to sepsis [14-19]. The test performed well in distinguishing infection from flare-ups in autoimmune disease [20-22], illustrating usefulness in the early detection of bacterial infection after surgery [23]. However, in patients with proven or suspected viral infection, neutrophil CD64 was also increased, though this increase was significantly lower than in patients with proven bacterial infections [24-
26]. In spite of the substantial research in recent years, there is no general view on the usability of neutrophil CD64 in clinical infection diagnostics [27-29]. Specifically, the diagnostic accuracy of CD64 in patients with AECOPD has not yet been studied.

The primary aim of this study was to determine the diagnostic accuracy of CD64 compared to the C-reactive protein (CRP) and white blood cell (WBC) count in identifying patients with pneumonia among hospitalized patients with AECOPD. Secondarily, we wanted to investigate the kinetics of CD64 expression during the first $48 \mathrm{~h}$ after admissions for AECOPD.

\section{Methods}

\section{Study Design}

The present study is a prospective, single-centre observation study.

\section{Participants}

Participants were prospectively recruited among patients hospitalized at the Department of Thoracic Medicine (DTM) of Trondheim University Hospital (TUH). The initial investigation of the patients was performed in the emergency department, and they were invited to participate in the study if the following criteria were met: (1) a clinical diagnosis of AECOPD; (2) an established diagnosis of COPD confirmed by spirometry results in the patients' medical charts; (3) the ability to give informed consent. The exclusion criteria were (1) a malignant disease; (2) bronchiectasis; (3) chronic bacterial colonization of the airways with Pseudomonas aeruginosa; (4) long-term immunosuppressive treatment; (5) long-term antibiotics treatment.

Patients admitted repeatedly were only included at their first admission. Pneumonia was defined as a new pulmonary infiltrate on chest X-ray at admission (PI). Patients who had not undergone chest X-ray at admission were not included in the analysis. Based on the findings of chest X-ray at admission, the patients were characterized into two groups: patients with evidence of AECOPD and PI (p-AECOPD) and those who had evidence of AECOPD without PI (npAECOPD). When the authors retrospectively reviewed the patients' medical charts, they were blinded to the CD64 results. The medical charts were reviewed for length of illness, symptoms, treatment with antibiotic or steroids within $48 \mathrm{~h}$ before admission and during the stay at the DTM, clinical parameters of the patients, CRP, WBC values and microbiological results as well as information about regular medication, unhealthy habits and pulmonary function results in a stable phase of COPD. 


\section{Informed Consent}

Informed consent was obtained from all participants included in the study.

\section{Sample Collection}

CRP and WBC counts were routinely analysed for all patients in the study, while additional blood samples (EDTA blood) for CD64 analysis were obtained at admission and 6, 24 and $48 \mathrm{~h}$ after admission. The standard hospital procedures for microbiological surveys were followed at blood sample collection and during the processing of biological materials (blood cultures and sputum).

\section{Laboratory Measurements}

CD64 expression was measured using the Leuko64 kit from Trillium Diagnostics, LLC (Maine, USA) with the CELL-DYN Sapphire Hematology System (Abbott Laboratories, Illinois, USA) and Leuko64TM QuantiCALC software from Trillium Diagnostics, LLC. The software calculated a CD64 index (CD64), which is supposed to be less than 1.00 in healthy individuals. Between-day coefficient of variation was $4.6 \%$ at a level of 2.61 .

WBC was measured on Sysmex XE-2100 (Sysmex, Kobe, Japan). The reference limits in adults were $3.7-10.0 \times 10^{9} / \mathrm{L}$, whereas the between-day coefficient of variation was $2.3 \%$ at $6.7 \times 10^{9} / \mathrm{L}$.

CRP was measured using a Roche modular $\mathrm{P}$ system (Roche Diagnostics GmbH, Mannheim, Germany), with reagents from the manufacturer and from Diagnostic Systems GmbH, Holzheim, Germany. The reference limit was less than $5.0 \mathrm{mg} / \mathrm{L}$, and the between-day coefficient of variation was $6.3 \%$ at $19 \mathrm{mg} / \mathrm{L}$.

All analyses were monitored using the appropriate internal and external (WBC and CRP) quality control systems.

All pulmonary function tests (spirometry) were performed using a MasterScreen PFT powered by SentrySuite $^{\mathrm{TM}}$ from Jaeger (Erich Jaeger GmbH, Würzburg, Germany), and COPD was defined as a post-bronchodilator $\mathrm{FEV}_{1} / \mathrm{FVC}<0.7$ according to the GOLD (2015) criteria.

The physicians who were in charge of the patients were responsible for the diagnostic procedures, laboratory analyses and all decisions regarding treatment and follow-up.

\section{Statistical Analysis}

The distribution of the continuous variables was studied using Q-Q plots, histograms, values of skewness and kurtosis, and normality was tested by the Kolmogorov-
Smirnov test. The median value (interquartile range) was used to present non-normal distributed variables. The Mann-Whitney $U$ test and Fishers exact test were used to compare quantitative and categorical data, respectively, in two groups. The Spearman rank correlation coefficient was used to study correlations. The Friedman test was used to examine differences between measurements repeated at several time points, while receiver operating characteristic (ROC) curve analysis was used to study the accuracy of the various diagnostic tests and logistic regression to find the best combination of diagnostic tests. $P$ values $<0.05$ were considered to be statistically significant. Statistical analyses were carried out through the use of computer IBM software SPSS 21 (Chicago, IL, USA) and StataCorp. 2011. Stata statistical software: Release 12. College Station, TX: StataCorp LP.

\section{Results}

From May 2011 to May 2013, a total of 159 patients were invited to participate in the study, with 113 patients meeting the inclusion criteria. A total of 36 (32\%) patients were classified as belonging to the p-AECOPD group, while 77 patients belonged to the np-AECOPD group. There were no significant differences in baseline characteristics between the patients in the two groups regarding age, lung function, proportion of current smokers, length of illness before admission or proportion of patients who received antibiotics and/or Prednisolone before admission. There was a higher proportion of males in the $\mathrm{p}$-AECOPD group compared with the np-AECOPD group (67 versus $33 \%, p<0.01$ ), and a larger proportion of patients were treated with antibiotics after admission in the p-AECOPD group compared with those in the np-AECOPD group (48 versus $92 \%, p<0.01)$, Table 1 .

Data on the CD64, CRP and WBC values were complete at admission in 98 patients, 31 (86 \%) in the p-AECOPD group versus $67(87 \%)$ in the np-AECOPD group. In the p-AECOPD group, the median values of CD64, CRP and WBC were statistically significantly higher than in the npAECOPD: 1.25 versus $0.60(p<0.01), 81.5$ versus 12.0 $(p<0.01)$ and 12.75 versus $9.6(p<0.01)$, respectively.

The diagnostic accuracy of the CD64, CRP and WBC in differentiating between the np-AECOPD and p-AECOPD was similar, with an area under the ROC curve of 0.69 (95\% CI 0.58-0.81) for CD64, 0.73 (95\% CI 0.62-0.84) for CRP and 0.64 (95\% CI 0.52-0.76) for WBC (Fig. 1). The differences were not statistically significant $(p=0.42)$. In a logistic regression model with CRP and WBC, CD64 did not reach statistical significance $(p=0.48)$. There was a strong positive correlation between CD64 and CRP, with rho $=0.64(p<0.01)$, i.e. 
Table 1 Demographic characteristics and disease status of the study population by groups

\begin{tabular}{|c|c|c|c|}
\hline \multirow[t]{2}{*}{ Characteristics } & \multicolumn{2}{|c|}{ Study population $(n=113)$} & \multirow{2}{*}{$\begin{array}{l}p \text { value } \mathrm{p}-\mathrm{AECOPD} \text { versus } \\
\text { np-AECOPD }\end{array}$} \\
\hline & p-AECOPD $(n=36)$ & np-AECOPD $(n=77)$ & \\
\hline Age & 68.0 & 71.0 & 0.4 \\
\hline Median (IQR) & $(65.0,76.0)$ & $(65.0,76.0)$ & \\
\hline Sex male, $n(\%)$ & $24(67)$ & $25(33)$ & 0.001 \\
\hline Current smokers, $n(\%)$ & $9(25.0)$ & $31(40.3)$ & 0.1 \\
\hline $\mathrm{FEV}_{1}$, median (IQR) & $0.79(0.56,1.19)$ & $0.74(0.52,0.93)$ & 0.4 \\
\hline $\mathrm{FEV}_{1} \%$, median (IQR) & $27(20,41)$ & $28(22,43)$ & 0.5 \\
\hline FVC, median (IQR) & $1.92(1.82,2.43)$ & $1.73(1.73,2.10)$ & 0.3 \\
\hline FVC \%, median (IQR) & $52(50,62)$ & $55(56,65)$ & 0.2 \\
\hline $\mathrm{FEV}_{1} / \mathrm{FVC}$, median (IQR) & $0.42(0.39,0.50)$ & $0.43(0.41,0.42)$ & 0.7 \\
\hline $\begin{array}{l}\text { Length of illness before hospitalization, days }(n) \text {, } \\
\text { median (IQR) }\end{array}$ & $6(3.0,7.8)$ & $7(1.5,12.0)$ & 0.9 \\
\hline \multicolumn{4}{|l|}{ Treatment before admission, $n(\%)$} \\
\hline Antibiotics & $10(28)$ & $18(23)$ & 0.6 \\
\hline Oral steroid & $12(33)$ & $26(34)$ & 1.0 \\
\hline \multicolumn{4}{|l|}{ Treatment after admission, $n(\%)$} \\
\hline Antibiotics & $33(92)$ & $37(48)$ & 0.001 \\
\hline Oral steroids & $32(89)$ & $68(88)$ & 1.0 \\
\hline Regular medication, combination ICS with LABA & $29(81)$ & $55(71)$ & 0.2 \\
\hline \multicolumn{4}{|l|}{ Blood parameters at admission, median (IQR) } \\
\hline WBC & $12.8(8.9,16.2)$ & $9.6(7.8,12.4)$ & 0.001 \\
\hline CRP & $81.5(18.3,163.5)$ & $12.0(5.0,50.0)$ & 0.001 \\
\hline CD64 & $1.25(0.70,1.95)$ & $0.60(0.5,1.0)$ & 0.002 \\
\hline
\end{tabular}

$A E C O P D$ acute exacerbations of chronic obstructive lung disease, $p$-AECOPD AECOPD with pulmonary infiltrate on chest X-ray at admission, $n p-A E C O P D$ AECOPD without pulmonary infiltrate on chest $\mathrm{X}$-ray at admission, $I Q R$ interquartile range, $F E V_{l}$ forced expiratory volume (litres) in one second, $F E V_{1} \% \mathrm{FEV}_{1}$ percent of predicted value, $F V C$ forced vital capacity (litres), $F V C \% \mathrm{FVC}$ percent of predicted value, $F E V_{l} / F V C$ $\mathrm{FEV}_{1}$ expressed as a proportion of FVC, ICS inhaled corticosteroids, $L A B A$ long-acting beta-adrenoceptor agonist, WBC white blood cell, $C R P$ C-reactive protein, CD64 neutrophil CD expression

high levels of CD64 were associated with high levels of CRP. Furthermore, there was no correlation between the CD64 and WBC values.

There was a complete data set on the CD64 at admission and 6, 24 and $48 \mathrm{~h}$ after admission in 25 patients in the p-AECOPD group and 49 patients in the np-AECOPD group (Table 2). At all test times, the CD64 values were higher in the patients with p-AECOPD than the patients with np-AECOPD, but only at admission did these differences reach statistical significance. In the p-AECOPD group, the CD64 values in blood samples taken at admission and $6 \mathrm{~h}$ after admission were statistically significantly higher than in samples taken 24 and $48 \mathrm{~h}$ after admission $(p<0.05)$. No such dependency on time was observed in np-AECOPD patients.

A blood culture sample was taken in 26 patients with npAECOPD and in 17 patients with p-AECOPD, but bacteremia was not present in any of the samples.

At admission, sputum samples were taken in 51 patients with np-AECOPD and in 19 patients with p-AECOPD.

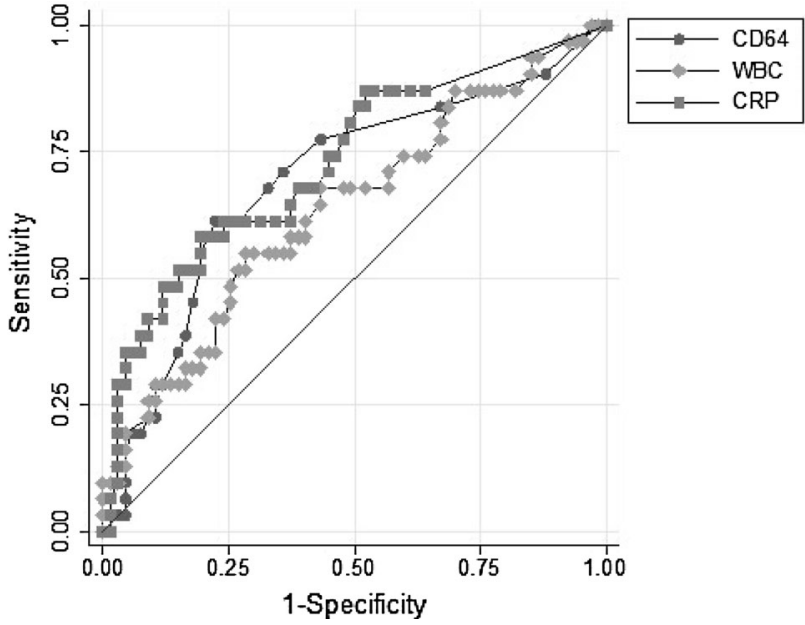

Fig. 1 Receiver operating characteristic (ROC) curves for CD64, WBC and CRP measured in samples taken at admission. The curves show how sensitivity (true positive fraction) varies with 1 -specificity (false positive fraction) when the diagnostic cut-off limit is varied 
Table 2 Median value of CD64 in blood samples taken at admission to the hospital, and 6, 24 and $48 \mathrm{~h}$ after admission, from patients with (p-AECOPD) and without (np-AECOPD) pulmonary infiltrate

\begin{tabular}{llll}
\hline $\begin{array}{l}\text { Time of sampling in hours } \\
\text { after admission }\end{array}$ & p-AECOPD $(n=25)$ & np-AECOPD $(n=49)$ & $\begin{array}{l}p \text { value p-AECOPD } \\
\text { versus np-AECOPD }\end{array}$ \\
\hline 0 & $1.30(0.60,2.05)^{\mathrm{a}}$ & $0.60(0.50,1.10)$ & 0.05 \\
6 & $1.20(0.50,2.20)^{\mathrm{a}}$ & $0.70(0.50,1.12)$ & 0.07 \\
24 & $0.90(0.50,2.25)$ & $0.70(0.50,1.30)$ & 0.20 \\
48 & $1.00(0.57,1.55)$ & $0.70(0.50,1.00)$ & 0.09 \\
\hline
\end{tabular}

$A E C O P D$ acute exacerbations of chronic obstructive lung disease, $p$-AECOPD AECOPD with pulmonary infiltrate on chest X-ray at admission, $n p-A E C O P D$ AECOPD without pulmonary infiltrate on chest $\mathrm{X}$-ray on admission

${ }^{a}$ Statistically significant higher values than measured in samples taken 24 and $48 \mathrm{~h}$ after admission. The numbers in parentheses are the corresponding interquartile ranges

Pathogens were found in 29 samples from the np-AECOPD group and in 10 samples from the p-AECOPD group. Individual CD64 values with corresponding findings in the sputum samples are shown in Table 3. H. influenza $(n=11), M$. catarrhalis $(n=5), S$. pneumonia $(n=6)$ and $S$. aureus $(n=3)$ were the agents most commonly found in the sputum samples. Mixed cultures, bacterial + bacterial $(n=5)$ and bacterial $+\operatorname{viral}(n=3)$ were found exclusively in samples from patients with npAECOPD. There was a great variability in the CD64 values between samples that were positive for the same pathogen.

\section{Discussion}

In the present study, we showed that the expression of CD64 on neutrophils is higher in patients with p-AECOPD than in patients with np-AECOPD, both at admission to the hospital and during the following $48 \mathrm{~h}$.

To the best of our knowledge, this is the first report on the diagnostic accuracy of the CD64 in identifying those with pneumonia among patients with AECOPD applied in a real clinical setting in which acutely ill patients were being examined. Two previous studies have covered some aspects of the CD64 expression in patients with AECOPD. In the study by Zhang and colleagues [30], the CD64 expression in patients with AECOPD and healthy subjects was compared, and in the study by Mao and colleagues [31] the CD64 expression in patients with AECOPD, patients with stable COPD and healthy subject were compared. The CD64 expression was higher in those with AECOPD than in patients with stable COPD. There was no difference in the CD64 expression between patients with stable COPD and healthy subjects. However, none of these studies evaluated the diagnostic accuracy of the CD64 to distinguish between p-AECOPD and np-AECOPD. In the present study, the elevated CD64 values in patients with p-AECOPD declined between 6 and $24 \mathrm{~h}$ after admission. However, it should be noted that $92 \%$ of the patients in the
p-AECOPD group received antibiotics, and changes in CD64 expression may be related to the antibiotic treatment through restoration of the regulated immune response by such treatments. This finding may indicate that measuring the CD64 expression might be useful in monitoring the effects of antibiotic therapy implying that CD64 could be used as a prognostic marker. The kinetics of the CD64 expression found in this study is supported by the findings of other researchers [23, 32].

The diagnostic accuracy of CD64 in differentiating between p-AECOPD and np-AECOPD was about the same as that of CRP. Furthermore, CD64 did not add to the diagnostic accuracy of CRP and WBC counts when these laboratory tests were combined to discriminate between p-AECOPD and np-AECOPD. It must be considered that these findings are partly due to some limitations of the present study. First, the study population is rather small, and therefore the confidence intervals of the ROC areas are relatively wide. The differences between CD64, CRP and WBC counts might have been statistically significant in a larger population. Second, some patients included in the study were treated with systemic steroids and/or antibiotics before admission. If all patients were left untreated until admission, the diagnostic accuracy might have been better for all the laboratory tests. Third, the study was not designed with regard to microbiological diagnostics. In a small number of patients, blood culture samples were requested by the physician in charge, but bacteremia was not present in any of the samples. Advanced microbiological analysis of sputum was not performed routinely. Hence, we were not able to correlate the CD64 levels with bacteriological or viral findings. Our data are consistent with results from previous studies regarding the association of $H$. influenzae, M. catarrhalis and S. pneumonia with AECOPD [33-36]. An interesting question that ought to be addressed in future studies is whether CD64 has the potential as a useful biomarker in distinguishing between an acute airway infection or colonization of the airways with one or more bacteria. 
Table 3 The individual values of neutrophil CD64 expression and positive culture findings
Recognized agents

Values of neutrophil CD64 expression

p-AECOPD $(n=10) \quad$ np-AECOPD $(n=29)$

Haemophilus influenzae

1.8

3.9 $1.1^{\mathrm{a}}$

$0.9^{\mathrm{c}}$

2.6

$0.5^{\mathrm{c}}$

1.6

0.5

Haemophilus influenzae plus

Klebsiella oxytoca

$0.9^{\mathrm{c}}$

Moraxella catarrhalis

0.7

Influenza A virus subtype H1N1

0.6

Moraxella catarrhalis

0.9

2.2

0.9

Moraxella catarrhalis plus

$\alpha$-haemolytic streptococcus

Stenotrophomonas maltophilia

Klebsiella pneumoniae

$\beta$-haemolytic Streptococcus group B

$\beta$-haemolytic Streptococcus group C plus Proteus species

Streptococcus pneumoniae

5.2

3.3

1.6

$1.5^{\mathrm{c}}$

Staphylococcus aureus

$0.4^{\mathrm{c}}$

$2.1^{\mathrm{b}}$

Staphylococcus aureus plus

$0.6^{\mathrm{c}}$

Coagulase-negative staphylococci

Stenotrophomonas maltophilia

$0.5^{\mathrm{b}}$

$1.8^{\mathrm{c}, \mathrm{d}}$

0.4

Proteus mirabilis + Rhinovirus PCR positive

$0.9^{\mathrm{c}}$

Influenza A virus subtype H1N1

$1.7^{\mathrm{c}}$

Enterobacter cloacae

$0.9^{\mathrm{c}}$

Candida albicans

1.1

$1.3^{\mathrm{c}}$

Acinetobacter baumannii

Gram-negative bacteria

a Treated with antibiotics before admission

b Treated with steroids per os before admission

c Treated with both steroids and antibiotics

d CD64 results from samples 6h after admission

$A E C O P D$ acute exacerbations of chronic obstructive lung disease, $p$-AECOPD AECOPD with pulmonary infiltrate on chest X-ray at admission, $n p-A E C O P D$ AECOPD without pulmonary infiltrate on chest X-ray at admission
The strength of the present study is the clinical validity of the study population prospectively included when admitted to hospital because of an AECOPD and the clinically relevant diagnostic procedure of p-AECOPD by chest X-rays without any knowledge of the value of CD64 or any other laboratory tests. 


\section{Conclusion}

When used for diagnosing pneumonia in patients with an acute exacerbations of COPD, CD64 had about the same diagnostic accuracy as CRP, and CD64 did not seem to add to the diagnostic accuracy of CRP and WBC counts.

Acknowledgments The authors would like to acknowledge the personnel of the Emergency Department, Departments of Thoracic Medicine, Clinical Chemistry and Medical Microbiology of the Trondheim University Hospital for their assistance. The authors would like to acknowledge Unimed Innovation AS, Trondheim University Hospital Foundation and the Research Council of Norway for partial support of this study.

\section{Compliance with Ethical Standards}

Conflict of Interest The authors declare that they have no financial or non-financial conflicts of interests.

Research Involving Human Participants All procedures performed in studies involving human participants were in accordance with the ethical standards of the Regional Committee for Medical and Health Research Ethics (REC) and with the 1964 Helsinki declaration and its later amendments or comparable ethical standards.

Open Access This article is distributed under the terms of the Creative Commons Attribution 4.0 International License (http://crea tivecommons.org/licenses/by/4.0/), which permits unrestricted use, distribution, and reproduction in any medium, provided you give appropriate credit to the original author(s) and the source, provide a link to the Creative Commons license, and indicate if changes were made.

\section{References}

1. Global Strategy for Diagnosis, Management, and Prevention of COPD Updated January (2015) Global initiative for chronic obstructive lung disease. http://www.goldcopd.org/. Accessed July 2015

2. Perera WR, Hurst JR, Wilkinson TM et al (2007) Inflammatory changes, recovery and recurrence at COPD exacerbation. Eur Respir J 29(3):527-534. doi:10.1183/09031936.00092506

3. Hurst JR, Perera WR, Wilkinson TM et al (2006) Systemic and upper and lower airway inflammation at exacerbation of chronic obstructive pulmonary disease. Am J Respir Crit Care Med 173(1):71-78. doi:10.1164/rccm.200505-704OC

4. Seemungal TA, Hurst JR, Wedzicha JA (2009) Exacerbation rate, health status and mortality in COPD - a review of potential interventions. Int J Chronic Obstr Pulm Dis 4:203-223

5. Wedzicha JA, Brill SE, Allinson JP et al (2013) Mechanisms and impact of the frequent exacerbator phenotype in chronic obstructive pulmonary disease. BMC Med 11:181. doi:10.1186/ 1741-7015-11-181

6. Seemungal T, Harper-Owen R, Bhowmik A et al (2001) Respiratory viruses, symptoms, and inflammatory markers in acute exacerbations and stable chronic obstructive pulmonary disease. Am J Respir Crit Care Med 164(9):1618-1623. doi:10.1164/ ajrccm.164.9.2105011

7. Wedzicha JA (2004) Role of viruses in exacerbations of chronic obstructive pulmonary disease. Proc Am Thorac Soc 1(2):115-120. doi:10.1513/pats.2306030
8. Papi A, Luppi F, Franco F et al (2006) Pathophysiology of exacerbations of chronic obstructive pulmonary disease. Proc Am Thorac Soc 3(3):245-251. doi:10.1513/pats.200512-125SF

9. Peacock JL, Anderson HR, Bremner SA et al (2011) Outdoor air pollution and respiratory health in patients with COPD. Thorax 66(7):591-596. doi:10.1136/thx.2010.155358

10. Quint JK, Donaldson GC, Goldring JJ et al (2010) Serum IP-10 as a biomarker of human rhinovirus infection at exacerbation of COPD. Chest 137(4):812-822. doi:10.1378/chest.09-1541

11. Hurst JR, Donaldson GC, Perera WR et al (2006) Use of plasma biomarkers at exacerbation of chronic obstructive pulmonary disease. Am J Respir Crit Care Med 174(8):867-874. doi:10. 1164/rccm.200604-506OC

12. Hoffmann JJ (2009) Neutrophil CD64: a diagnostic marker for infection and sepsis. Clin Chem Lab Med 47(8):903-916. doi:10. 1515/cclm.2009.224

13. Bakke AC, Allen E, Purtzer MZ et al (2001) Neutrophil CD64 expression distinguishing acute inflammatory autoimmune disease from systemic infections. Clin Appl Immunol Rev 1(5):267-275

14. Davis BH, Olsen SH, Ahmad E et al (2006) Neutrophil CD64 is an improved indicator of infection or sepsis in emergency department patients. Arch Pathol Lab Med 130(5):654-661. doi:10.1043/1543-2165(2006)130[654:nciaii]2.0.co;2

15. Livaditi O, Kotanidou A, Psarra A et al (2006) Neutrophil CD64 expression and serum IL-8: sensitive early markers of severity and outcome in sepsis. Cytokine 36(5-6):283-290. doi:10.1016/j. cyto.2007.02.007

16. Cardelli P, Ferraironi M, Amodeo R et al (2008) Evaluation of neutrophil CD64 expression and procalcitonin as useful markers in early diagnosis of sepsis. Int $\mathrm{J}$ Immunopathol Pharmacol 21(1):43-49

17. Farias MG, de Lucena NP, Dal Bo S et al (2014) Neutrophil CD64 expression as an important diagnostic marker of infection and sepsis in hospital patients. J Immunol Methods 414:65-68. doi:10.1016/j.jim.2014.07.011

18. Gamez-Diaz LY, Enriquez LE, Matute JD et al (2011) Diagnostic accuracy of HMGB-1, sTREM-1, and CD64 as markers of sepsis in patients recently admitted to the emergency department. Acad Emerg Med 18(8):807-815. doi:10.1111/j.1553-2712.2011.01113.x

19. Hsu KH, Chan MC, Wang JM et al (2011) Comparison of Fcgamma receptor expression on neutrophils with procalcitonin for the diagnosis of sepsis in critically ill patients. Respirology 16(1):152-160. doi:10.1111/j.1440-1843.2010.01876.x

20. Doi T, Miyazaki T, Nishino J et al (2010) Neutrophil CD64 expression as a diagnostic marker for local infection and crystalinduced arthritis. Mod Rheumatol 20(6):573-579. doi:10.1007/ s10165-010-0322-6

21. Matsui T, Ohsumi K, Ozawa N et al (2006) CD64 on neutrophils is a sensitive and specific marker for detection of infection in patients with rheumatoid arthritis. J Rheumatol 33(12):24162424

22. Allen E, Bakke AC, Purtzer MZ et al (2002) Neutrophil CD64 expression: distinguishing acute inflammatory autoimmune disease from systemic infections. Ann Rheum Dis 61(6):522-525

23. Fjaertoft G, Hakansson LD, Pauksens K et al (2007) Neutrophil CD64 (FcgammaRI) expression is a specific marker of bacterial infection: a study on the kinetics and the impact of major surgery. Scand J Infect Dis 39(6-7):525-535. doi:10.1080/00365540601113693

24. Rudensky B, Sirota G, Erlichman M et al (2008) Neutrophil CD64 expression as a diagnostic marker of bacterial infection in febrile children presenting to a hospital emergency department. Pediatr Emerg Care 24(11):745-748. doi:10.1097/PEC. Ob013e31818c2679

25. Jalava-Karvinen P, Hohenthal U, Laitinen I et al (2009) Simultaneous quantitative analysis of Fc gamma RI (CD64) and CR1 
(CD35) on neutrophils in distinguishing between bacterial infections, viral infections, and inflammatory diseases. Clin Immunol 133(3):314-323. doi:10.1016/j.clim.2009.08.003

26. Fjaertoft G, Pauksen K, Hakansson L et al (2005) Cell surface expression of Fc gamma RI (CD64) on neutrophils and monocytes in patients with influenza $\mathrm{A}$, with and without complications. Scand J Infect Dis 37(11-12):882-889. doi:10.1080/ 00365540500348929

27. Nuutila $\mathbf{J}$ (2010) The novel applications of the quantitative analysis of neutrophil cell surface FcgammaRI (CD64) to the diagnosis of infectious and inflammatory diseases. Curr Opin Infect Dis 23(3):268-274. doi:10.1097/QCO.0b013e32833939b0

28. Cid J, Aguinaco R, Sanchez R et al (2010) Neutrophil CD64 expression as marker of bacterial infection: a systematic review and meta-analysis. J Infect 60(5):313-319. doi:10.1016/j.jinf. 2010.02.013

29. Li S, Huang X, Chen Z, Zhong H et al (2013) Neutrophil CD64 expression as a biomarker in the early diagnosis of bacterial infection: a meta-analysis. Int J Infect Dis 17(1):e12-e23. doi:10. 1016/j.ijid.2012.07.017

30. Zhang LX, Ye J, Chen YB et al (2013) The effect of CD33 expression on inflammatory response in chronic obstructive pulmonary disease. Immunol Investig 42(8):701-710. doi:10.3109/ 08820139.2013.806542
31. Mao W, Jiang Y, Ouyang L et al (2014) Early diagnostic values of CD64 and CD11b indices of peripheral white blood cells for acute exacerbation of chronic obstructive pulmonary disease in older adults. Xi Bao Yu Fen Zi Mian Yi Xue Za Zhi 30(11):1201-1204

32. Barth E, Fischer G, Schneider EM et al (2001) Differences in the expression of CD64 and mCD14 on polymorphonuclear cells and on monocytes in patients with septic shock. Cytokine 14(5):299-302. doi:10.1006/cyto.2001.0880

33. Rosell A, Monso E, Soler N et al (2005) Microbiologic determinants of exacerbation in chronic obstructive pulmonary disease. Arch Intern Med 165(8):891-897. doi:10.1001/archinte. 165.8.891

34. Miravitlles M, Espinosa C, Fernandez-Laso E et al (1999) Relationship between bacterial flora in sputum and functional impairment in patients with acute exacerbations of COPD. Study Group of Bacterial Infection in COPD. Chest 116(1):40-46

35. Patel IS, Seemungal TA, Wilks M et al (2002) Relationship between bacterial colonisation and the frequency, character, and severity of COPD exacerbations. Thorax 57(9):759-764

36. Barker BL, Haldar K, Patel $\mathrm{H}$ et al (2015) Association between pathogens detected using quantitative polymerase chain reaction with airway inflammation in COPD at stable state and exacerbations. Chest 147(1):46-55. doi:10.1378/chest.14-0764 